\title{
Complexation of semiflexible chains with oppositely charged cylinder
}

\author{
Andrey G. Cherstvy a) and Roland G. Winkler \\ Institut für Festkörperforschung, Theorie-II, Forschungszentrum Jülich, D-52425 Jülich, Germany
}

(Received 14 November 2003; accepted 24 February 2004)

\begin{abstract}
We study the complexation of long thin semiflexible polymer chains with an oppositely charged cylinder. Starting from the linear Poisson-Boltzmann equation, we calculate the electrostatic potential and the energy of such a charge distribution. We find that sufficiently flexible chains prefer to wrap around the cylinder in a helical manner, when their charge density is smaller than that of the cylinder. The optimal value of the helical pitch is found by minimization of the sum of electrostatic and bending energies. The dependence of the pitch on the number of chains, their rigidity, and salt concentration in solution is analyzed. We discuss our results in the light of recent experiments on DNA complexation with cylindrical dendronized polymers. (C) 2004 American Institute of Physics.
\end{abstract} [DOI: $10.1063 / 1.1707015]$

\section{INTRODUCTION}

Compactification of DNA into $\sim 100$-nm large complexes with oppositely charged proteins and polycations is an important tool in gene therapy ${ }^{1}$ to deliver DNA into an infected cell. ${ }^{2}$ In particular, DNA condensates with polylysine $^{3,4}$ and polyamines ${ }^{5,6}$ are used for these purposes. Nucleosome core particles and supercoiled DNA's are also fundamentally important biological objects which involve a DNA arrangement in a helical manner. Although the complexation and aggregate formation of chains of various flexibilities and of different lengths with oppositely charged spheres and cylinders has been studied theoretically, ${ }^{7-16}$ experimentally, ${ }^{4,17-22}$ and by computer simulations, ${ }^{23-30} \mathrm{sev}-$ eral features of this phenomenon are still not completely understood.

One of these features is overcharging of a cylinder by adsorbed oppositely charged chains predicted theoretically ${ }^{15,16}$ and recently reported experimentally for cylindrical DNA/dendrimer complexes. ${ }^{19}$ In contrast, DNA wrapped around spherical dendrimers was shown to form preferentially neutral complexes. ${ }^{18,21,22}$ Nature also uses similar techniques to compact the genetic material in cells, where the nucleosome can be substantially overcharged by a DNA molecule wrapped around it. ${ }^{31,32}$ Another aspect is the theoretically predicted release of a chain from a sphere at high salt concentrations ${ }^{8}$ that reminds salt-induced DNA release from the nucleosome observed experimentally. ${ }^{33,34} \mathrm{In}$ experiments in vitro at higher salt concentrations, it has been reported that DNA attaches stronger to cylindrical dendrimers ${ }^{20}$ but weaker to spherical ones. ${ }^{21}$ The physical origin of this effect is not clear yet.

In many cases, electrostatic interactions play a dominant role in the formation of complexes between highly charged macromolecules. Wrapping of DNA duplexes around a cylindrical/spherical object is, however, a formidable electrostatic problem, which can involve chiral interactions between DNA helices. ${ }^{35}$ The details and discreteness of charge distri-

${ }^{a)}$ Corresponding author. Electronic mail: a.cherstvy@fz-juelich.de bution on DNA might also be important (DNA overwinding from $10.5 \mathrm{bp} /$ turn in solution ${ }^{36}$ to about $10.2 \mathrm{bp} /$ turn in nucleosomes ${ }^{31,32,37}$ occurs). Large charge densities of both objects introduce additional complications, since the linear Poisson-Boltzmann theory may not apply. However, to understand the basic physical properties of complexes, it might be sufficient to start from the simplest model.

In this article we consider the wrapping of thin semiflexible charged chains around an oppositely charged cylinder. We calculate the electrostatic potential and energy of the helical complex using linear Poisson-Boltzmann theory. Hence, our calculations extend those of Ref. 12, because the full solution of the linear Poisson-Boltzmann equation is considered for the particular charge distribution and not simply a superposition of Debye-Hückel potentials. This has consequences for the amount of DNA adsorbed on a cylinder. Within our model neutral and undercharged complexes are preferred, whereas the calculations of Ref. 12 predict a significant overcharging. We compare the predictions of the model with available theoretical and experimental data. In the end we discuss some possible extensions of the model.

The paper is organized as follows. In Sec. II the model is outlined and the electrostatic energy for a helical charge distribution on a cylinder is calculated. In Sec. III the electrostatic energy for a helical charge distributions is determined. Results for the helical pitch are presented and discussed in Sec. IV. Finally, Sec. V summarizes our findings.

\section{ENERGY CALCULATION}

\section{A. Model and approximations}

We solve the linear Poisson-Boltzmann equation for an infinitely long, positively charged cylinder with negatively charged semiflexible strings adsorbed on its surface in a helical conformation. The strings are considered to be infinitely thin charged lines. No fluctuations of the strings on the cylinder surface are considered (zero-temperature solution, the helices are ideal). The cylinder has the radius $a$ and the surface charge density $\sigma_{c}$ (the linear charge density is $e_{0} \tau_{c}, e_{0}$ is the elementary charge). For simplicity, no distribution of 
the electrostatic potential inside the cylinder is considered. Every string is characterized by a linear charge density $e_{0} \tau_{p}$ and the mechanical persistence length $l_{p}$. The water is considered as a dielectric continuum (no discreteness effects) with the dielectric constant $\varepsilon \approx 80$. The dielectric constant of the cylinder interior is the same as in the bulk solution (no image forces). No effects of charge fluctuations and correlations are considered.

\section{B. Electrostatic energy}

The electrostatic energy of such a charge distribution follows from the electrostatic potential $\phi(\vec{r})$ with the charge density $\rho(\vec{r})$ according to

$$
\begin{aligned}
E_{e l} & =\frac{1}{2} \int d^{3} \vec{r} \phi(\vec{r}) \rho(\vec{r}) \\
& =\frac{a}{2} \int_{0}^{2 \pi} d \varphi \int_{-\infty}^{\infty} d z \phi(z, \varphi, a) \sigma(z, \varphi) .
\end{aligned}
$$

The volume charge density $\rho$ is related to the surface charge density $\sigma$ of the complex via $\rho(z, \varphi, r)=\delta(r-a) \sigma(z, \varphi)$. Cylindrical coordinates are used, where the $z$ axis is oriented along the axis of the cylinder. Below, we consider a helicallike distribution of charges that suggests using a Fourier representation of the potential and of the surface charge density,

$$
\left\{\begin{array}{c}
\phi(z, \varphi, r) \\
\sigma(z, \varphi)
\end{array}\right\}=\int_{-\infty}^{\infty} d k e^{i k z} \sum_{n=-\infty}^{\infty} e^{i n \varphi}\left\{\begin{array}{c}
\widetilde{\phi}(k, n, r) \\
\widetilde{\sigma}(k, n)
\end{array}\right\}
$$

with

$$
\begin{aligned}
\left\{\begin{array}{c}
\widetilde{\phi}(k, n, r) \\
\widetilde{\sigma}(k, n)
\end{array}\right\}= & \frac{1}{(2 \pi)^{2}} \int_{-\infty}^{\infty} d z e^{-i k z} \int_{0}^{2 \pi} d \varphi e^{-i n \varphi} \\
& \times\left\{\begin{array}{c}
\phi(z, \varphi, r) \\
\sigma(z, \varphi)
\end{array}\right\} .
\end{aligned}
$$

Note that $\sigma(z, \varphi)$ in these relations may have no helical symmetry. In terms of Fourier components of the electrostatic potential $\widetilde{\phi}(k, n, r)$, and of the surface charge density $\widetilde{\sigma}(k, n)$, the general energy expression is

$$
E_{e l}=(2 \pi)^{2} \frac{a}{2} \int_{-\infty}^{\infty} d k \sum_{n=-\infty}^{\infty} \widetilde{\phi}(k, n, a) \widetilde{\sigma}(-k,-n) .
$$

\section{Electrostatic potential}

We now consider the complex (cylinder plus wrapped semiflexible strings) in an electrolyte solution with the Debye screening length $\kappa^{-1}$. We calculate the electrostatic potential $\phi(z, \varphi, r)$ created by the charge distribution $\sigma(z, \varphi)$ using the linearized $\left(\left|e_{0} \phi\right| \ll k_{B} T\right)$ Poisson-Boltzmann equation

$$
\frac{\partial^{2} \phi}{\partial r^{2}}+\frac{1}{r} \frac{\partial \phi}{\partial r}+\frac{1}{r^{2}} \frac{\partial^{2} \phi}{\partial \varphi^{2}}+\frac{\partial^{2} \phi}{\partial z^{2}}=\kappa^{2} \phi .
$$

Applying the Fourier transformation, the equation for $\widetilde{\phi}(k, n, r)$ reads

$$
\begin{aligned}
& \frac{d^{2} \widetilde{\phi}(k, n, r)}{d\left(\tilde{\boldsymbol{\kappa}}_{k} r\right)^{2}}+\frac{1}{\tilde{\boldsymbol{\kappa}}_{k} r} \frac{d \widetilde{\phi}(k, n, r)}{d\left(\tilde{\boldsymbol{\kappa}}_{k} r\right)}-\left(\frac{n^{2}}{\left(\tilde{\boldsymbol{\kappa}}_{k} r\right)^{2}}+1\right) \widetilde{\phi}(k, n, r) \\
& \quad=0
\end{aligned}
$$

where $\tilde{\kappa}_{k}=\sqrt{\kappa^{2}+k^{2}}$. This is the differential equation for the modified Bessel functions of the second kind. Its general solution is

$$
\widetilde{\phi}(k, n, r)=\alpha K_{n}\left(\widetilde{\kappa}_{k} r\right)+\beta I_{n}\left(\tilde{\kappa}_{k} r\right),
$$

where the constants $\alpha$ and $\beta$ are to be found from the boundary conditions. When the cylinder is inserted into a cylindrical cell of the radius $R_{s}$, the boundary conditions on the surface of the cylinder (Gauss theorem) and on the surface of the cell are

$$
\left.\frac{d \widetilde{\phi}(k, n, r)}{d\left(\tilde{\kappa}_{k} r\right)}\right|_{r=R_{s}}=0,\left.\quad \frac{d \widetilde{\phi}(k, n, r)}{d\left(\tilde{\kappa}_{k} r\right)}\right|_{r=a}=-\frac{4 \pi \widetilde{\sigma}(k, n)}{\varepsilon \tilde{\kappa}_{k}} .
$$

The general solution for $\widetilde{\phi}(k, n, r)$ satisfying the boundary conditions (8) is

$$
\begin{aligned}
\widetilde{\phi}(k, n, r)= & -\frac{4 \pi \widetilde{\sigma}(k, n)}{\varepsilon \tilde{\kappa}_{k}} \\
& \times \frac{I_{n}^{\prime}\left(\tilde{\kappa}_{k} R_{s}\right) K_{n}\left(\tilde{\kappa}_{k} r\right)-K_{n}^{\prime}\left(\tilde{\kappa}_{k} R_{s}\right) I_{n}\left(\tilde{\kappa}_{k} r\right)}{I_{n}^{\prime}\left(\tilde{\kappa}_{k} R_{s}\right) K_{n}^{\prime}\left(\tilde{\kappa}_{k} a\right)-I_{n}^{\prime}\left(\tilde{\kappa}_{k} a\right) K_{n}^{\prime}\left(\tilde{\kappa}_{k} R_{s}\right)} .
\end{aligned}
$$

For a uniformly charged cylinder the surface charge is $\sigma(z, \varphi)=-\sigma_{c}>0$ and hence $\widetilde{\sigma}(k, n)=-\sigma_{c} \delta(k) \delta_{n, 0}$, where $\delta(k)$ is the Dirac delta function and $\delta_{n, m}$ is the Kronecker delta ( $\delta_{n, m}=1$ if $n=m$ and $\delta_{n, m}=0$ otherwise). The electrostatic potential is then of the form $e_{0} \phi_{0}(r) /\left(k_{B} T\right)$ $=2 \xi K_{0}(\kappa r) /\left[\kappa a K_{1}(\kappa a)\right]$, where $l_{B}=e_{0}^{2} /\left(\varepsilon k_{B} T\right)$ is the Bjerrum length and $\xi=l_{B} \tau_{c}$ is the well-known polyelectrolyte charge density parameter $\left(1 / \tau_{c}\right.$ is the separation between unit charges along the cylinder axis).

For a complex in an infinite volume $\left(R_{s} \rightarrow \infty\right)$, Eq. (9) yields

$$
\widetilde{\phi}(k, n, r)=-\frac{4 \pi a \widetilde{\sigma}(k, n)}{\varepsilon} \frac{K_{n}\left(\tilde{\kappa}_{k} r\right)}{\tilde{\kappa}_{k} a K_{n}^{\prime}\left(\tilde{\kappa}_{k} a\right)} .
$$

Since the fixed charges are located on the cylindrical surface, the energy depends only on the surface potential. Equation (4) then gives the general energy expression

$$
E_{e l}=-a(2 \pi)^{3} \int_{-\infty}^{\infty} d k \sum_{n=-\infty}^{\infty} \frac{|\widetilde{\sigma}(k, n)|^{2}}{\varepsilon \tilde{\kappa}_{k}} \frac{K_{n}\left(\tilde{\kappa}_{k} a\right)}{K_{n}^{\prime}\left(\tilde{\kappa}_{k} a\right)}
$$

in terms of $|\widetilde{\sigma}(k, n)|^{2}$ for arbitrary helical charge pattern.

\section{HELICES}

In this section we determine the charge densities $\widetilde{\sigma}(k, n)$ and the energies for an adsorbed double helix on a neutral cylinder as well as for a double helix and multihelix adsorbed on the surface of an oppositely charged cylinder. The 
first two results can be used to study certain electrostatic properties of the double-stranded DNA. The third distribution is used in the present work to calculate the energy of a complex of many polyelectrolyte chains adsorbed on the surface of an oppositely charged cylinder in a helical manner. As cylinder charges are distributed homogeneously on its surface, we speak further about the "jellium" helices (for DNA, for instance, the smeared out charge of adsorbed cations forms the cylinder charge).

\section{A. Double helix}

We model a double helix by two negatively charged helical strings on the cylinder surface, with a mean surface charge density $\sigma_{p}$. The helices are right-handed with the helical pitch $H>0$ and are separated by the distance $h$ along the axis of the cylinder. The charge distribution $\sigma(z, \varphi)$ for the infinitely long helix ${ }^{38}$ is given by is given by

$$
\begin{aligned}
\sigma(z, \varphi)= & \pi \sigma_{p} \sum_{m}\{\delta(\varphi+2 \pi m-g z) \\
& +\delta[\varphi+2 \pi m-g(z+h)]\},
\end{aligned}
$$

where $g=2 \pi / H$. The Fourier coefficients of the charge density (12) are given by

$$
\widetilde{\sigma}(k, n)=\sigma_{p} \delta(k+n g)[1+\exp (-i n g h)] / 2 .
$$

Using the Fourier coefficients (10) and Eq. (2) the electrostatic potential created by a charge distribution $\sigma(z, \varphi)$ is the sum of harmonics,

$$
\frac{e_{0} \phi(z, \varphi, r)}{k_{B} T}=2 \xi_{p} \frac{K_{0}(\kappa r)}{\kappa a K_{1}(\kappa a)}-2 \xi_{p} \sum_{n=1}^{\infty} \frac{\{\cos [n(\varphi-g z)]+\cos [n(\varphi-g z)-n g h]\} K_{n}\left(\kappa_{n} r\right)}{\kappa_{n} a K_{n}^{\prime}\left(\kappa_{n} a\right)},
$$

where $\xi_{p}=l_{B} \eta \tau_{p}$ and $\eta=\sqrt{1+(2 \pi a / H)^{2}}$. The renormalized reciprocal screening length for $n$th harmonic is

$$
\kappa_{n}=\sqrt{\kappa^{2}+n^{2}(2 \pi / H)^{2}} .
$$

The term $n=0$ in Eq. (14) corresponds to the potential $\phi_{0}(r)$ of a uniformly charged cylinder. The terms with $n$ $\neq 0$ are "corrections," which reflect the helicity of the charge distribution. These potential terms vary along the helix and may produce an accumulation of mobile cations in the vicinity of the negatively charged helical strings (cf. Fig. 1).

By evaluation of the integral of Eq. (11), we obtain the electrostatic self-energy $E_{e l}$ per pitch (linear energy density) as the sum of harmonics,

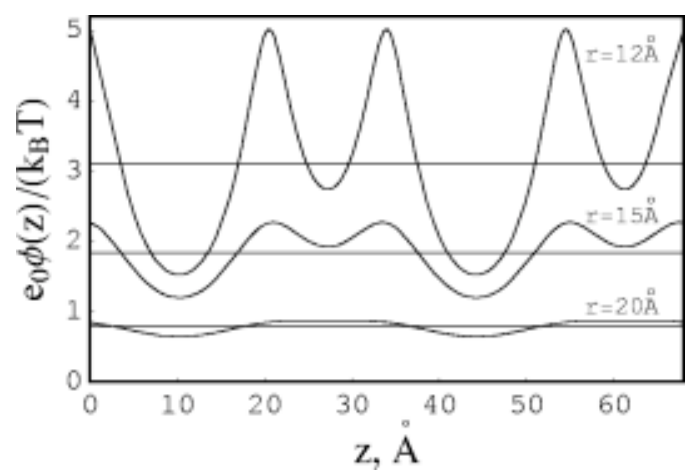

FIG. 1. Electrostatic potential of a double helix with $B$-DNA parameters, with no adsorbed cations, according to Eq. (14) with $\varphi=0$ at physiological salt concentration. The potential variation decreases with increasing separation from the molecular axis increases (lines indicate the potential of the corresponding uniformly charged cylinder). If the counterion condensation is taken into account, the potential variation along the molecule will be less than unity, and the linear Poisson-Boltzmann theory becomes strictly valid.

$$
\begin{aligned}
E_{e l}= & \frac{4 \pi^{2} \sigma_{p}^{2} a^{2}}{\varepsilon}\left\{\frac{K_{0}(\kappa a)}{\kappa a K_{1}(\kappa a)}\right. \\
& \left.-\sum_{n=1}^{\infty} \frac{[1+\cos (n g h)] K_{n}\left(\kappa_{n} a\right)}{\kappa_{n} a K_{n}^{\prime}\left(\kappa_{n} a\right)}\right\}>0 .
\end{aligned}
$$

Term with $n=0$ corresponds to the self-energy of a uniformly charged cylinder with surface charge density $\sigma_{p}$. The terms with $n \neq 0$ again are corrections to this energy caused by the helicity of the charged strings. $E_{e l}[$ Eq. (16)] has a minimum at $h=H / 2$, where the electrostatic repulsion between the helical strings is minimal. When $H$ decreases, $\kappa_{n}$ increase and the electrostatic interaction becomes effectively better screened. Thus the sum in Eq. (16) favors $H \rightarrow 0$. The value of each term in the sum decreases with $\kappa$ and $a$, since the function $K_{n}(\kappa a) /\left[-\kappa a K_{n}^{\prime}(\kappa a)\right]$ decays with $\kappa a$.

In the limit $h \rightarrow 0$, we obtain the energy density for a single strand,

$$
E_{e l}=\frac{4 \pi^{2} \sigma_{p}^{2} a^{2}}{\varepsilon}\left\{\frac{K_{0}(\kappa a)}{\kappa a K_{1}(\kappa a)}-2 \sum_{n=1}^{\infty} \frac{K_{n}\left(\kappa_{n} a\right)}{\kappa_{n} a K_{n}^{\prime}\left(\kappa_{n} a\right)}\right\} .
$$

This corresponds to the self-energy of the helix considered in Ref. 12. The energy terms are, however, different. The authors of Ref. 12 find $E_{e l} \sim \tau_{p}^{2} \eta(\eta-1)$, whereas our solution of the Poisson-Boltzmann equation yields $E_{e l} \sim \tau_{p}^{2} \eta^{2}$. This difference has severe consequences on the charge of the cylinder and on the possibility of its overcharging by adsorbed helices, as we will discuss in the next section.

\section{B. Double-stranded jellium helix}

We now consider two negatively charged helical strings with a helical pitch $H$ adsorbed on the surface of a positively charged cylinder. The charge density reads 


$$
\begin{aligned}
\sigma(z, \varphi)= & -\sigma_{c}+\pi \sigma_{p} \sum_{m}\{\delta(\varphi+2 \pi m-g z) \\
& +\delta[\varphi+2 \pi m-g(z+h)]\},
\end{aligned}
$$

and $\quad \tilde{\sigma}(k, n)=-\sigma_{c} \delta(k) \delta_{n, 0}+\sigma_{p} \delta(k+g n)[1+\exp$ $(-i n g h)] / 2$. The electrostatic energy density of the charge distribution (18) is given by

$$
\begin{aligned}
E_{e l}= & \frac{4 \pi^{2} \sigma_{c}^{2} a^{2}}{\varepsilon}\left\{\frac{K_{0}(\kappa a)}{\kappa a K_{1}(\kappa a)}(1-\theta)^{2}\right. \\
& \left.-\theta^{2} \sum_{n=1}^{\infty} \frac{[1+\cos (n g h)] K_{n}\left(\kappa_{n} a\right)}{\kappa_{n} a K_{n}^{\prime}\left(\kappa_{n} a\right)}\right\},
\end{aligned}
$$

where we introduced the ratio

$$
\theta=\frac{\sigma_{p}}{\sigma_{c}}=\frac{\eta \tau_{p}}{\tau_{c}} .
$$

The repulsion between the strings disfavors full compensation of the cylinder charge by wrapped strings, and the complex is usually undercharged. In the limit $h \rightarrow 0$, the energy density of a single string is obtained. Finally, $E_{e l}$ decreases nearly exponentially with $\kappa$ at large $\kappa a$.

The molecule with the charge distribution (18) can be considered as a model for a $B$-DNA helix: charges on the strands represent the phosphates and cylinder charge corresponds to adsorbed cations, smeared on the DNA surface (for $B$-DNA: $\left.{ }^{32} a \approx 10 \AA, h \approx 0.4 H\right) .{ }^{39}$

\section{Multistranded jellium helix}

The model for two strands can easily be extended to a situation with many adsorbed strands. For $N_{s}$ helical negatively charged strands, equally separated on the surface of a positively charged cylinder, the charge distribution is given by

$$
\begin{aligned}
\sigma(z, \varphi)= & -\sigma_{c}+\pi \sigma_{p} \sum_{m} \sum_{s=0}^{N_{s}-1} \delta[\varphi+2 \pi m \\
& \left.-g\left(z+s H / N_{s}\right)\right] .
\end{aligned}
$$

Here $\sigma_{p}$ denotes the mean surface charge density for each string and $H / N_{s}$ is the separation between the strings along the cylinder axis. If the total charge density of the strings $N_{s} \sigma_{p}=2 \sigma_{c}$, they totally compensate the charge of the cylinder. The electrostatic energy density of the charge density (20) is given by

$$
\begin{aligned}
E_{e l}= & \frac{4 \pi^{2} \sigma_{c}^{2} a^{2}}{\varepsilon}\left\{\frac{K_{0}(\kappa a)}{\kappa a K_{1}(\kappa a)}(1-\theta)^{2}\right. \\
& \left.-\theta^{2} \sum_{j=1}^{\infty} \frac{2 K_{j N_{s}}\left(\kappa_{j N_{s}} a\right)}{\kappa_{j N_{s}} a K_{j N_{s}}^{\prime}\left(\kappa_{j N_{s}} a\right)}\right\} .
\end{aligned}
$$

The product $j N_{s}$ gives the index of the functions $K_{n}\left(\kappa_{n} a\right)$. For $N_{s}=2$, the expression (21) turns into Eq. (19) [only the terms with even $n$ survive in Eq. (19) at $h=H / 2]$. The energy density for a single string follows for $N_{s}=1$. This corresponds to the situation considered in Ref. 12. However, the expressions for the total energy of the cylinder+helices are different (cf. Sec. III B). This has consequences for the effective charge of the complex.

The first term in Eq. (21) vanishes when the strings fully compensate the charge of the cylinder. Thus this term favors a finite value of $H$, i.e., a helical conformation of the strings. The second term (the sum) represents the repulsive interaction among the strings. The sum is always negative, because $K_{n}^{\prime}$ is negative. Hence this term will lead to a larger pitch the one given by the first term alone. As a consequence, the complex will at best be neutral or its effective number density of charges, $\tau_{\text {eff }}=\tau_{c}-\eta \tau_{p}$, will exhibit the same sign as the cylinder, in contrast to the findings in Ref. 12.

The convergence of the series in energy expressions (16), (19), and (21) depends on the ratio $\kappa /(2 \pi / H)$. If the argument of the Bessel function $\kappa_{n} a$ grows substantially with $n$, the $n$th term in the sum (16) decreases rapidly with $n$. Thus, for relatively large $H(\kappa \gg 2 \pi / H)$, the contribution to $\kappa_{n} a$ with increasing $n$ is small and the convergence of the series is slow. In the opposite limit $(\kappa \ll 2 \pi / H)$, the convergence is fast. Thus, with increase of $H$ the summation becomes less accurate and we have to take into account more terms in the sum to achieve the same precision. The convergence of the series is better for a larger number of adsorbed strings, $N_{s}$ (at the same $H$ ).

\section{Bending energy}

The mechanical bending energy $\left(E_{\text {bend }}\right)$ associated with the rigidity of a string disfavors the helical conformation, which is favored by charge neutralization of the complex. Larger persistence lengths $l_{p}$ and smaller cylinder radii $a$ result in a higher bending energy for the wrapped conformation. Parametrization of the radius vector $\vec{r}(s)$ along the helix, $\vec{r}(s)=\{a \cos [2 \pi s /(\eta H)], a \sin [2 \pi s /(\eta H)], s / \eta\}^{T}$, yields for the energy density along the cylinder with $N_{s}$ wrapped strings,

$$
\begin{aligned}
E_{\text {bend }}(H) & \approx N_{s} k_{B} T \frac{l_{p}}{2} \lim _{l \rightarrow \infty} \frac{1}{l} \int_{-l / 2}^{+l / 2}\left(\frac{\partial^{2} \vec{r}(s)}{\partial s^{2}}\right)^{2} d s \\
& =N_{s} k_{B} T \frac{l_{p}}{2 a^{2}} \frac{(2 \pi a / H)^{4}}{\left[1+(2 \pi a / H)^{2}\right]^{3 / 2}},
\end{aligned}
$$

where $|d \vec{r}(s) / d s|=1$. This energy is the same as used in Ref. 12. Other effects, which can affect the wrapping of the chains, are considered in the next section.

\section{RESULTS AND DISCUSSION}

\section{A. Linear Poisson-Boltzmann approach}

The optimal helical pitch of the complex $H$ is found by minimizing the total-energy density

$$
\begin{aligned}
E\left(\tau_{p}, \tau_{c}, \kappa, N_{s}, H, a\right)= & E_{e l}\left(\tau_{p}, \tau_{c}, \kappa, N_{s}, H, a\right) \\
& +E_{\text {bend }}\left(H, a, l_{p}, N_{s}\right) .
\end{aligned}
$$

Figure 2 shows results for $H$ at various string numbers $N_{s}$ in the case of constant surface charge density $\theta=1$ of the strings and of the cylinder. As expected, the optimal $H$ value increases with increasing chain stiffness. The optimal $H$ also 


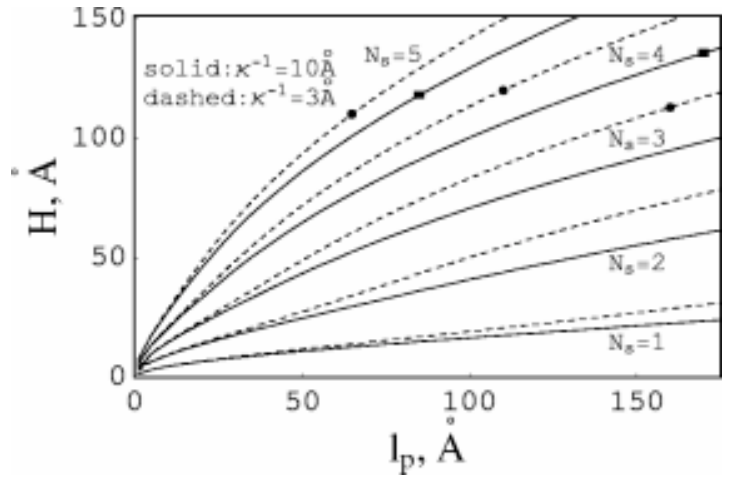

FIG. 2. Optimal helical pitch of the complex with $N_{s}$ equidistant strings as a function of their persistence length for the parameters $\theta=1, a=10 \AA$, and $\sigma_{c}=\sigma_{B-\mathrm{DNA}}=16.8 \mu \mathrm{C} / \mathrm{cm}^{2}$ [Eq. (21) is used]. The dots on the curves denote the points with an energy depth of $0.1 k_{B} T / \AA$, compared to the $H$ $\rightarrow \infty$ state.

increases with $N_{s}$ because of the higher total bending energy ${ }^{40}$ For larger $\kappa$ the optimal pitch increases since the electrostatic interaction is more efficiently screened and the same bending energy leads to an energy minimum at larger $H$ (dashed lines in Fig. 2).

For constant linear charge densities $\tau_{p}$ and $\tau_{c}$ and for weakly charged chains (when $\tau_{p} \ll \tau_{c}$ ), the electrostatic energy has a minimum at a finite value of $H$ and thus the helical conformation is favored for small $N_{s}$ values. Larger persistence lengths $l_{p}$ disfavor wrapping (Fig. 3). Consequently, a smaller fraction of the cylinder charge $\theta=\sigma_{p} / \sigma_{c}$ $=\eta \tau_{p} / \tau_{c}$ is compensated by wrapped chains (cf. Fig. 3). The pitch increases with $N_{s}$ and with $l_{p}$ (Fig. 3). For given
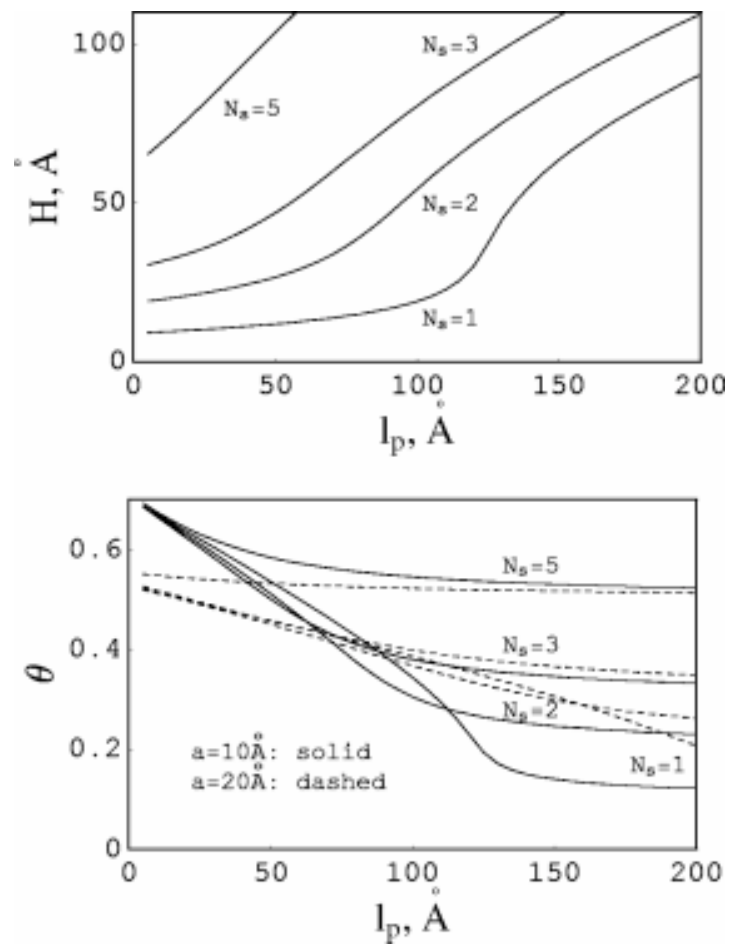

FIG. 3. Optimal helical pitch of the complex and its charge neutralization fraction $\theta$ for constant $\tau_{p, c}$ and for various chain numbers. Parameters: $a$ $=10 \AA$ and $a=20 \AA, \kappa=1 /\left(20 \AA\right.$ ) , $\tau_{c}=1 / \AA$, and $\tau_{p}=0.1 / \AA$ (the cylinder is ten times more charged than each string).
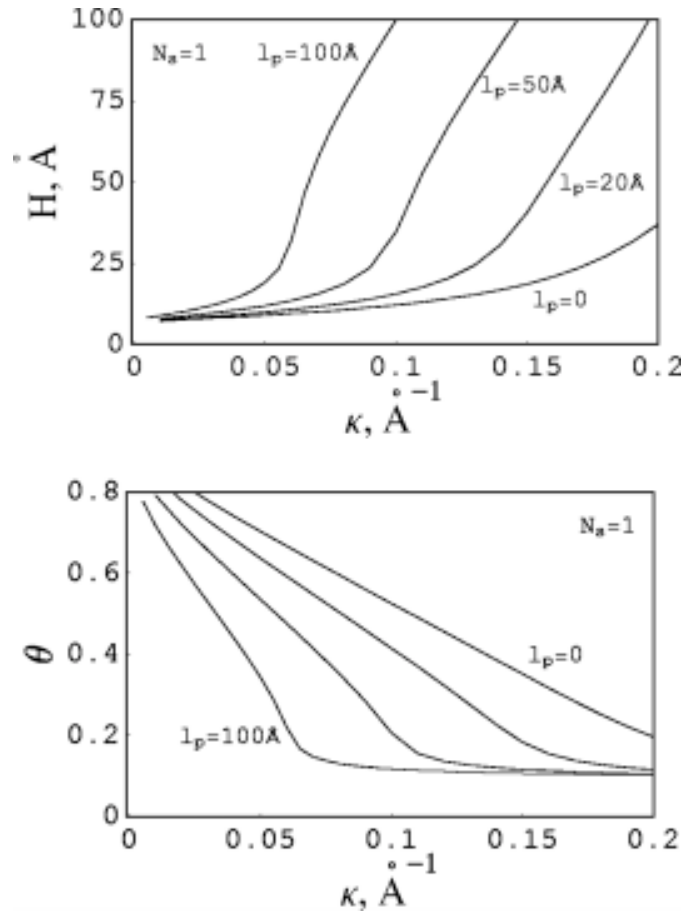

FIG. 4. Optimal helical pitch of the complex and its charge neutralization fraction as a function of $\kappa$ for $N_{s}=1$ and for several values of the persistence length $l_{p}$. Other parameters are the same as in Fig. 3 .

$\tau_{p}$ and $\tau_{c}$ and beyond a certain number of adsorbed strings, the energy difference between a helical conformation and a conformation with straight rods is rather small. Hence we may observe both kinds of conformations, in particular when we take thermal fluctuations of strings into account. This applies to the system with $N_{s}>5$ in Fig. 3. Vice versa, for a given $N_{s}$ there is a threshold ratio $\tau_{p} / \tau_{c}$ above which the straight conformation is almost as favorable as the helical one.

With an increase of the cylinder radius, the corresponding electrostatic energy decreases for fixed linear charge densities. That may lead to higher values of the helical pitch (although $E_{\text {bend }}$ decreases) and to a weaker charge neutralization of the cylinder by wrapped strings (Fig. 3). With decreasing linear charge densities, the value of the helical pitch increases more rapidly with the persistence length. Please note that in Fig. 3 such large $\tau_{p}$ and $\tau_{c}$ values are used that the linear Poisson-Boltzmann theory is no longer strictly applicable. But, it will provide an estimation of the features following from the full theory. With increasing salt concentration, the value of the optimal helical pitch increases for weakly charged chains (Fig. 4). At small $\kappa$, the electrostatic interaction between string and the cylinder is strong enough to compensate a large portion of the cylinder charge, whereas with increasing $\kappa$ the neutralization fraction $\theta$ decreases rapidly. Figure 4 shows this dependence for $N_{s}=1$ and for several values of the persistence length.

Our theory agrees with experiments on DNA/dendrimer complexes which display an increase of the pitch with decreasing dendrimer charge density. ${ }^{19}$ Theoretical predictions of Ref. 12 also indicate that the straight conformation of a single string adsorbed on an oppositely charged cylinder be- 
comes favorable at high salt concentrations. Our model also shows that for a fixed number of strings $N_{s}$ the optimal $H$ increases when $\kappa$ increases (cf. Fig. 2), because $E_{e l}$ decreases and the energy minimum shifts to larger $H$. In experiments, the pitch of DNA wrapping around dendrimers indeed increases with $\kappa$ for spherical ${ }^{21}$ dendrimers, but it decreases for cylindrical dendrimers. ${ }^{20,41}$ One might argue that the DNA persistence length decreases at high salt concentrations, because the electrostatic ${ }^{42}$ persistence length $l_{p, e l}=l_{B} /\left(4 \kappa^{2} b_{p}^{2}\right)$ decreases. The DNA could then wrap denser around the cylinder. However, such effects should occur for spherical complexes also, but have not been observed in experiments.

Another unresolved issue is the charge of DNA/ dendrimer complexes. Preferentially neutral complexes of DNA with spherical dendrimers were observed in experiments of Refs. 21 and 22, in contrast to a pronounced (up to two times) overcharging of cylindrical dendrimers by wrapped DNA reported in Ref. 19. The adsorption/attraction of cylindrical complex onto a positively charged silica surface was used as an evidence for overcharging in Ref. 19. To have this attraction, however, the overcharging may not be required, since the negative DNA charges are closer to the silica surface than the positive charges of the dendrimer. Hence advanced experiments are required to understand the nature of a possible overcharging of cylindrical DNA/ dendrimer complexes.

\section{B. Extensions of the model}

We summarize below a few possible extensions of the model.

(i) In the main text the adsorption of chains directly on the cylinder surface was considered. At nonzero temperature this is entropically unfavorable. For instance, the free energy penalty upon confinement of the chain of the length $L \gg l_{p}$ within the cylinder of diameter $\delta$ is proportional to $L$, $F_{\text {conf }} \sim k_{B} T\left(L / l_{p}\right) \ln \left(l_{p} / \lambda\right)$. Here $\lambda=l_{p}^{1 / 3} \delta^{2 / 3}$ is the so-called deflection length ${ }^{46}$ (see also Ref. 47). The minimization of $E_{e l}+F_{\text {conf }}$ would then lead to an optimal thickness of the layer of adsorbed polymer. Like for the polyelectrolyte adsorption on the plane, ${ }^{48}$ the chain may start to adsorb on the cylinder only at $\sigma_{c}>\sigma_{c}^{t h}$. The entropic repulsion between the chains within the layer is expected to become more pronounced with $\kappa$, like for DNA adsorption on a plane. ${ }^{49}$ Another entropic effect is the counterion release upon adsorption of a polymer chain on an oppositely charged macroion. ${ }^{10,16}$ This occurs when the polymer is not directly on the cylinder surface. Its quantitative investigation requires the full calculation of the ion distribution around the polymer and the cylinder, ${ }^{50}$ before and after the polymer adsorption.

(ii) We considered infinitely long chains and the mobile ions were assumed to ensure screening only, with no adsorption onto the surface of the cylinder. In reality, the polymer chains may have a finite length and the mobile cations can also be adsorbed on the surface of a highly charged macroion. Then the coverage should be determined selfconsistently, like in the problem of competitive adsorption in electrochemistry. ${ }^{51}$ A proper free-energy functional can be constructed on the basis of the Flory theory. ${ }^{52}$ (iii) Several mechanisms of macroion overcharging by oppositely charged polymers were suggested in the literature. ${ }^{10,12,15,16,53}$ One mechanism, which is relevant for adsorption on curved surfaces, suggests that the adsorption of highly charged semiflexible chains of finite radius around a cylinder may be accompanied by charge neutralization along their contact (like for DNA wrapping around the histone core in the nucleosome ${ }^{32}$ ). Then excess charges on the outer chain surface appear, and their electrostatic repulsion produces a spontaneous curvature of the chain towards the cylinder. This would change the calculated helical pitch and may even lead to an overcharging of the complex.

\section{CONCLUSIONS}

In the present article, we have analytically calculated the energy of the helical conformation of charged strings on the surface of an oppositely charged cylinder and the dependence of the helical pitch on the model parameters. We have calculated the electrostatic potential created by a helical charge distribution and the energy of the system in such a potential. The present work extends the treatment of Ref. 12 for an arbitrary number of helical strings. We have also shown that the transition from a helical to a straight conformation is a continuous transition, rather than an abrupt one. In our pure energy minimum solution we do not observe the pronounced overcharging of the complex, reported in Ref. 12 on the basis of a superposition solution for interaction energies. We rather find neutral or undercharged complexes. We currently extend this Poisson-Boltzmann treatment to spherical complexes. The results will be reported elsewhere.

\section{ACKNOWLEDGMENTS}

We are thankful to I. Gössl, M. Castelnovo, A. A. Kornyshev, S. V. Malinin, and R. R. Netz for many useful discussions.

${ }^{1}$ V. Vijayanathan, T. Thomas, and T. J. Thomas, Biochemistry 41, 14085 (2002), and references therein.

${ }^{2}$ J. F. Kukowska-Latallo, A. U. Belinska, J. Johnson, R. Spindler, D. A. Tomalia and J. R. Baker, Jr. Proc. Natl. Acad. Sci. U.S.A. 93, 4897 (1996).

${ }^{3}$ H. G. Hansma, R. Golan, W. Hsieh, C. P. Lollo, P. Mullenley, and D. Kwoh, Nucleic Acids Res. 26, 2481 (1998).

${ }^{4}$ U. K. Laemmli, Proc. Natl. Acad. Sci. U.S.A. 72, 4288 (1975).

${ }^{5} \mathrm{~V}$. Vijayanathan, T. Thomas, A. Shirahata, and T. J. Thomas, Biochemistry 40, 13644 (2001).

${ }^{6}$ J. A. Schellman and N. Parthasarathy, J. Mol. Biol. 175, 313 (1984).

${ }^{7}$ R. R. Netz and J.-F. Joanny, Macromolecules 32, 9026 (1999).

${ }^{8}$ K.-K. Kunze and R. R. Netz, Phys. Rev. E 66, 011918 (2002).

${ }^{9}$ H. Schiessel, R. F. Bruinsma, and W. M. Gelbart, J. Chem. Phys. 115, 7245 (2001)

${ }^{10}$ E. M. Mateescu, C. Jeppesen, and P. Pincus, Europhys. Lett. 46, 493 (1999)

${ }^{11}$ H. Schiessel, J. Phys.: Condens. Matter 15, R699 (2003).

${ }^{12}$ K.-K. Kunze and R. R. Netz, Europhys. Lett. 58, 299 (2002).

${ }^{13}$ R. R. Netz, Physik Journal 1, 51 (2002).

${ }^{14}$ T. Odijk, Macromolecules 13, 1542 (1980).

${ }^{15}$ T. T. Nguyen, A. Yu. Grosberg, and B. I. Shklovskii, J. Chem. Phys. 113, $1110(2000)$.

${ }^{16}$ S. Y. Park, R. F. Bruinsma, and W. M. Gelbart, Europhys. Lett. 46, 454 (1999).

${ }^{17}$ J. X. Tang, T. Ito, T. Tao, P. Traub, and P. A. Jamney, Biochemistry 36, 12600 (1997).

${ }^{18}$ A. U. Bielinska, C. Chen, J. Johnson, and J. R. Baker, Jr., Bioconjugate Chem. 10, 843 (1999). 
${ }^{19}$ I. Gössl, L. Shu, D. Schlüter, and J. P. Rabe, J. Am. Chem. Soc. 124, 6860 (2002).

${ }^{20}$ I. Gössl, L. Shu, D. Schlüter, and J. P. Rabe, Single Mol. 3, 315 (2002).

${ }^{21}$ W. Chen, N. J. Turro, and D. A. Tomalia, Langmuir 16, 15 (2000).

${ }^{22}$ V. A. Kabanov, V. G. Sergeyev, O. A. Pyshkina, A. A. Zinchenko, A. B. Zezin, J. G. H. Joosten, J. Brackman, and K. Yoshikawa, Macromolecules 23, 9587 (2000).

${ }^{23}$ R. G. Winkler, M. O. Steinhauser, and P. Reineker, Phys. Rev. E 66, 021802 (2002)

${ }^{24}$ R. G. Winkler, New J. Phys. 6, 11 (2004).

${ }^{25}$ Y. Hayashi, M. Ullner, and P. Linse, J. Chem. Phys. 116, 6836 (2002).

${ }^{26}$ M. Skepö and P. Linse, Macromolecules 36, 508 (2003); F. Carlsson, M. Malmsten, and P. Linse, J. Am. Chem. Soc. 125, 3140 (2003).

${ }^{27}$ P. Chodanowski and S. Stoll, J. Chem. Phys. 115, 4951 (2001); T. Wallin and P. Linse, ibid. 109, 5089 (1998).

${ }^{28}$ M. Skepö and P. Linse, Phys. Rev. E 66, 051807 (2002).

${ }^{29}$ R. Messina, J. Chem. Phys. 119, 8133 (2003).

${ }^{30}$ A. Akinchina and P. Linse, J. Phys. Chem. B 107, 8011 (2003).

${ }^{31}$ K. Luger, A. M. Mäder, R. K. Richmond, D. F. Sangent, and T. J. Richmond, Nature (London) 389, 251 (1997).

${ }^{32}$ W. Saenger, Principles of Nucleic Acid Structure (Springer-Verlag, New York, (1984).

${ }^{33}$ T. D. Yager, C. T. McMurray, and K. E. van Holde, Biochemistry 28, 2271 (1989).

${ }^{34}$ A. Leforestier, S. Fudaley, and F. Livolant, J. Mol. Biol. 290, 481 (1999).

${ }^{35}$ A. A. Kornyshev, S. Leikin, and S. V. Malinin, Eur. Phys. J. E 7, 83 (2002).

${ }^{36}$ D. Rhodes and A. Klug, Nature (London) 286, 573 (1980); S. B. Zimmerman and B. H. Pheiffer, Proc. Natl. Acad. Sci. U.S.A. 76, 2703 (1979).

${ }^{37}$ M. Levitt, Proc. Natl. Acad. Sci. U.S.A. 75, 640 (1978).

${ }^{38}$ The charge distribution for the helix of a finite length $L$ is given by $\left.\sigma(z, \varphi)=\pi \sigma_{p} \Sigma_{m}\{\delta(\varphi+2 \pi m-g z)+\delta[\varphi+2 \pi m-g(z+h))]\right\} \Theta(L / 2$ $-z) \Theta(L / 2+z)$, where $\Theta(x)$ is the unit Heaviside step function. The Fourier coefficient of this charge density is $\widetilde{\sigma}(k, n)=2 \pi \sigma_{p}(2 \pi)^{-2}(1$ $\left.+e^{-i n g h}\right) \sin [(k+n g) L / 2] /(k+n g)$. This expression allows us to calculate the potential of the helix with a finite length. In the limit $L \rightarrow \infty$ using $\lim _{L \rightarrow \infty}[\sin (q L / 2) / q]=\pi \delta(q)$, we get of course the same energy of the complex, Eq. (16).

${ }^{39}$ Note that Eq. (19) could also be used for the description of electrostatically induced conformational changes of DNA, e.g., for the $B$ - to $Z$-DNA transition at high salt concentration. Note also that the exact theory of electrostatic interaction between two DNA duplexes predicts an attraction between them due to zipperlike charge separation along the molecules [A. A. Kornyshev and S. Leikin, Phys. Rev. Lett. 82, 4138 (1999)]. The interaction energy is the sum of harmonics that reflects the helicity of the charge distibution; the effective screening length of $n$th harmonic is $\kappa_{n}^{-1}$.

${ }^{40}$ Note that the calculation of the optimal number of strings $N_{s}$ and the part of the string attached to the cylinder (cylinder and strings are of finite sizes) requires the full free energy of charged semiflexible chains in solution which is a more complicated task.

${ }^{41}$ The helical pitch in salt-free solution of DNA wrapped around a cylindrical dendrimer was reported to be $\sim 23 \pm 3 \AA$ (Ref. 19). This is smaller than the pitch of DNA in a nucleosome, 24-27 $\AA$ (Refs. 31 and 32), which is close to the equilibrium DNA-DNA separation in hexagonal aggregates (Refs. 43 and 44) and in 2D DNA lattices (Ref. 45). Note that in the region of interaxial separations $\sim 20-25 \AA$ a very strong DNA-DNA repulsion is detected (Ref. 43). The pitch of DNA-dendrimer complexes was reported to further decrease at higher salt concentrations (Ref. 20). A simultanious swelling of the dendrimers, however, complicates the calculation of the real fraction of the neutralized dendrimer charge by the wrapped DNA.

${ }^{42}$ J. Skolnick and M. Fixman, Macromolecules 10, 944 (1977).

${ }^{43}$ D. C. Rau and V. A. Parsegian, Biophys. J. 61, 260 (1992).

${ }^{44}$ A. G. Cherstvy, A. A. Kornyshev, and S. Leikin, J. Phys. Chem. B 106, 13362 (2002)

${ }^{45}$ I. Koltover, K. Wagner, and C. R. Safinya, Proc. Natl. Acad. Sci. U.S.A. 97, 14046 (2000); J. O. Rädler, I. Koltover, T. Salditt, and C. R. Safinya, Science 275, 810 (1997).

${ }^{46}$ T. Odijk, Macromolecules 16, 1340 (1983).

${ }^{47}$ O. V. Borisov, E. B. Zhulina, and T. M. Birshtein, J. Phys. II 4, 913 (1994).

${ }^{48}$ R. R. Netz and J.-F. Joanny, Macromolecules 32, 9013 (1999).

${ }^{49}$ Y. Fang and J. Yang, J. Phys. Chem. B 101, 441 (1997).

${ }^{50}$ H. Ohshima, Colloid Polym. Sci. 274, 1176 (1996); 277, 563 (1999).

${ }^{51}$ J. M. Parry and R. Parsons, J. Electrochem. Soc. 113, 992 (1966); B. B. Damaskin, A. N. Frumkin, and N. A. Borovaya, Soviet Electrochemistry 8, 807 (1972).

${ }^{52}$ P. J. Flory and G. Ronca, Mol. Cryst. Liq. Cryst. 54, 289 (1979).

${ }^{53}$ W. M. Gelbart, R. F. Bruinsma, P. A. Pincus, and V. A. Parsegian, Phys. Today 53, 38 (2000), and references therein. 